\title{
How to select Tyrosine Kinase Inhibitor for the patients with newly diagnosed Chronic Myeloid Leukemia?
}

\author{
Nakaya A*, Fujita S, Satake A, Nakanishi T, Azuma Y, Tsubokura Y, Konishi A, Hotta M, Yoshimura H, \\ Ishii K, Ito T and Nomura $S$
}

First Department of Internal Medicine, Kansai Medical University, Osaka, Japan

*Corresponding author: Nakaya A, First Department of Internal Medicine, Kansai Medical University, 2-5-1, Shin-machi, Hirakata, Osaka 573-1010, Japan, Tel: +81-72-804-2503, E-mail: nakaya1016@yahoo.co.jp

Citation: Nakaya A, Fujita S, Satake A, Nakanishi T, Azuma Y, et al. (2018) How to select Tyrosine Kinase Inhibitor for the patients with newly diagnosed Chronic Myeloid Leukemia? J Hematol Blood Disord 4(1): 103. doi: $10.15744 / 2455-7641.4 .103$

Received Date: June 8, 2018 Accepted Date: August 15, 2018 Published Date: August 17, 2018

\begin{abstract}
The clinical outcomes of chronic myeloid leukemia (CML) have been improved by tyrosine kinase inhibitors (TKIs). However, there is no established consensus for TKI selection in de novo CML. We investigated TKI treatment patterns in a real-world setting. Among 95 chronic-phase CML patients, $44 \%$ were initiated treatment with imatinib, $26 \%$ with dasatinib, and 30\% with nilotinib. Our data suggest that imatinib remains applicable and that dasatinib has a favorable therapeutic effect, although pleural effusion can arise. Nilotinib was the most prevalent TKI as the treatment-associated adverse events were deemed more manageable than those associated with imatinib and dasatinib.
\end{abstract}

Keywords: Chronic Myeloid Leukemia; Tyrosine Kinase Inhibitor; Imatinib; Dasatinib; Nilotinib

\section{Introduction}

Chronic myeloid leukemia (CML) is a hematological myeloproliferative disorder characterized by the Philadelphia chromosome and its product, BCR-ABL, a constitutively active tyrosine kinase. The introduction of the first tyrosine kinase inhibitor (TKI) imatinib, dramatically improved the treatment of CML, with an overall survival (OS) of approximately $90 \%$ at 5 years [1].

In the IRIS study, a multicenter, randomized study of interferon versus imatinib for CML, $28 \%$ of patients discontinued therapy, and the main reasons for discontinuation were adverse events (AEs) or an unsatisfactory therapeutic effect [2]. The proportion of CML patients that acquire drug resistance in the chronic phase is generally low $(<25 \%)$ [3]. However, once patients develop resistance, poor clinical outcomes follow, with an OS of only $50 \%$ at 5 years [3]. However, patients exhibiting primary resistance or a suboptimal response to first-line TKI therapy within 12 months of treatment initiation may benefit from switching to another TKI [4]. Switching is also an option for patients who experience intolerable AEs. The subsequent selection of second-line therapy may depend on types of AEs or on specific mutations in BCR-ABL [5].

Adherence rates may also differ between clinical trials and real-world settings. Some studies have found that in an observational or a real-world setting, at least one-quarter of patients may not be adherent to TKI therapy, and that non-adherence can lead to a suboptimal response or disease relapse [6]. Some factors associated with poor adherence to TKIs include AEs, dose reduction, prolonged lag time between diagnosis and therapy initiation, and a high number of concomitant medications [7].

To date, no head-to-head trial comparing TKIs for the first-line treatment of CML has been reported. Thus, there is no established consensus for drug selection, which is currently at the discretion of the treating physician. The aim of the present study was to investigate the use of TKI therapy for de novo chronic-phase CML (CP-CML) in a real-world setting in Japan.

\section{Materials and Methods}

A total of 95 consecutive patients diagnosed with CML-CP and treated with TKI as initial therapy at Kansai Medical University Hospital from January 2002 to December 2015 were included in this retrospective study. 
CML diagnosis followed established diagnostic criteria. The treatment strategy for CML-CP was based on the decision of each treating physician's decision and considered CML sub-classification, age, performance status, complications, and patient's decision regarding therapy.

Between January 2002 and March 2009, only the first-generation TKI imatinib $400 \mathrm{mg}$ once daily was indicated as first-line treatment for CML-CP in Japan. After March 2009, the second-generation TKIs dasatinib $100 \mathrm{mg}$ once daily and nilotinib $300 \mathrm{mg}$ twice daily were approved for use as first-line treatment. Efficacy was assessed according to the European Leukemia Net (ELN) 2006 definition.

Response monitoring was performed by standard blood counts and bone marrow examination.

Molecular response was assessed by quantitative reverse-transcriptase PCR and converted to the International Scale (IS).

The severity of adverse events was graded using the National Cancer Institute Common Terminology Criteria for Adverse Events, version 4.0. This retrospective analysis was approved by the Ethics Committee of Kansai Medical University.

\section{Results}

\section{Patient characteristics}

The median age of the patients was 61 years (range, 19-89 years), and 61\% were male. We used three major risk stratification scales to predict the outcome: Sokal score, Hasford score, and EUTOS score. However, most patients were classified as having low-risk CML according to the Sokal and EUTOS scores (70\% and 84\%, respectively) and low- and intermediate-risk CML according to the Hasford score (low, 27\%; intermediate, $73 \%$ ) (Table 1).

\begin{tabular}{|l|c|}
\hline No. of patients (n) & 95 \\
\hline Median age, range (y/o) & $61(19-89)$ \\
\hline Male sex (\%) & 61 \\
\hline Sokal score (\%) & \\
\hline Low & 70 \\
\hline Intermediate & 28 \\
\hline High & 2 \\
\hline Hasford score (\%) & \\
\hline Low & 27 \\
\hline Intermediate & 73 \\
\hline High & 2 \\
\hline EUTOS score (\%) & \\
\hline Low & 84 \\
\hline High & 16 \\
\hline First-line therapy (\%) & \\
\hline Imatinib & 44 \\
\hline Dasatinib & 26 \\
\hline Nilotinib & 30 \\
\hline Second-line therapy (\%) & \\
\hline Imatinib & 5 \\
\hline Dasatinib & 15 \\
\hline Nilotinib & 80 \\
\hline
\end{tabular}

Table 1: Patients' characteristics

\section{TKI treatment flow}

Patients who were diagnosed before $2009(n=36)$ were started with imatinib as first-line treatment. After 2009, 6 patients were started with imatinib as first-line treatment. Among the 42 patients in total who were started with imatinib, 14 switched to a second-line TKI, of which 2 received dasatinib and 12 received nilotinib (Figure 1).

After March 2009, 25 patients started with dasatinib as first-line treatment and 5 of these patients subsequently switched to another TKI (imatinib, $\mathrm{n}=1$; nilotinib, $\mathrm{n}=4$ ). During this period, 28 patients started with nilotinib as first-line treatment but only one of these patients switched to another TKI (dasatinib).

Overall, as second-line treatment, imatinib was administered to one patient, dasatinib to 3 patients, and nilotinib to 16 patients. 


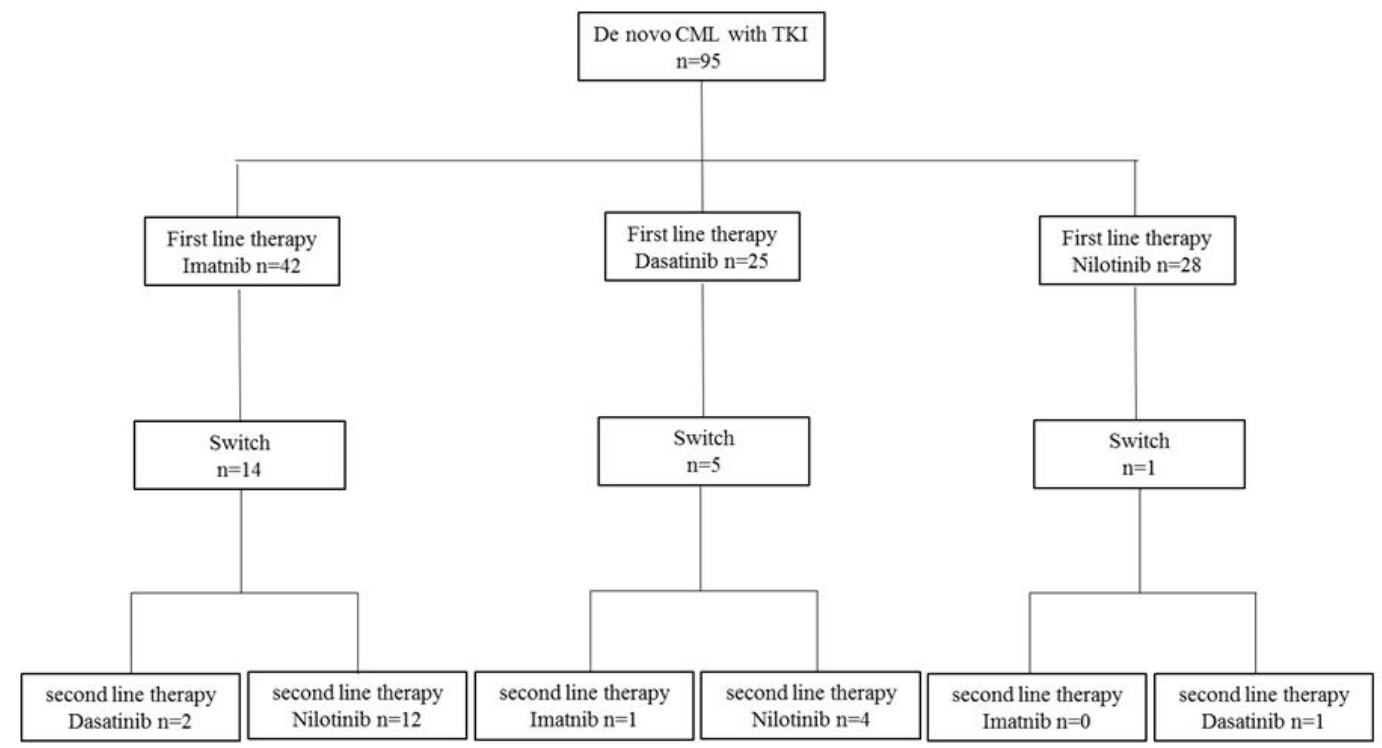

CML: Chronic Myeloid Leukemia; TKI: Tyrosine Kinase Inhibitor

In total, 95 consecutive patients diagnosed with CML-CP treated with TKI as initial therapy. 42 patients were started with imatinib for the first line. Among them, 14 patients switched to second-line TKI; two patients were to dasatinib and twelve patients were to nilotinib.

25 patients started with dasatinib as the first-line treatment. Among them, 5 patients were switched to another TKI. One patient was to imatinib and four patients were to nilotinib. On the other hand, 28 patients were started with nilotinib. Among them, only one patient was switched to dasatinib.

Figure 1: Treatment flow of TKI

\section{Adverse events and responses}

The rate of dose reduction because of AEs was 50\% with imatinib, $40 \%$ with dasatinib, and $21 \%$ with nilotinib. Patients who were worse adherence were $12 \%$ with imatinib, $4 \%$ with dasatinib, and $4 \%$ with nilotinib. Contents of the types of adverse events $(\geq$ grade 3) differed for each TKI, with edema most frequently observed with imatinib treatment (10\%), pleural effusion with dasatinib (16\%), and thrombocytopenia with nilotinib (11\%). TKI switching because of AEs was $29 \%$ for imatinib, $60 \%$ for dasatinib, and $100 \%$ for nilotinib, while switching because of treatment failure was $71 \%$ for imatinib, $40 \%$ for dasatinib, and $0 \%$ for nilotinib. More than $70 \%$ of all patients, regardless of the type of TKI, achieved a deep major molecular response (MMR). Failure to achieved MMR was observed in $17 \%$ of patients receiving imatinib, $12 \%$ receiving dasatinib, and $0 \%$ receiving nilotinib (Table 2 ).

\begin{tabular}{|c|c|c|c|c|c|c|}
\hline & \multicolumn{2}{|c|}{ Imatinib $(n=42)$} & \multicolumn{2}{|c|}{ Dasatinib $(n=25)$} & \multicolumn{2}{|c|}{ Nilotinib $(n=28)$} \\
\hline Reduced dose (\%) & \multicolumn{2}{|l|}{50} & \multicolumn{2}{|l|}{40} & \multicolumn{2}{|l|}{21} \\
\hline Worse adherence (\%) & \multicolumn{2}{|l|}{12} & \multicolumn{2}{|l|}{4} & \multicolumn{2}{|l|}{4} \\
\hline Switch (\%) & \multicolumn{2}{|l|}{33} & \multicolumn{2}{|l|}{20} & \multicolumn{2}{|l|}{4} \\
\hline \multirow[t]{2}{*}{ Reason for switch (\%) } & adverse events & 29 & adverse events & 60 & adverse events & 100 \\
\hline & failure & 71 & failure & 40 & failure & 0 \\
\hline \multirow[t]{3}{*}{ Adverse Events (\%) } & edema & 17 & $\begin{array}{l}\text { pleural } \\
\text { effusion }\end{array}$ & 16 & thrombocytopenia & 11 \\
\hline & neutopenia & 7 & rash & 8 & liver dysfunction & 7 \\
\hline & $\begin{array}{l}\text { interstitial } \\
\text { pneumonia }\end{array}$ & 5 & $\begin{array}{c}\text { QT } \\
\text { prolongation }\end{array}$ & 4 & pancreatitis & 4 \\
\hline \multirow[t]{5}{*}{ Response(\%) } & failure & 17 & failure & 12 & failure & 0 \\
\hline & CHR & 7 & $\mathrm{CHR}$ & 4 & $\mathrm{CHR}$ & 18 \\
\hline & CCyR & 0 & CCyR & 0 & CCyR & 11 \\
\hline & MMR & 42 & MMR & 52 & MMR & 39 \\
\hline & CMR & 33 & CMR & 32 & CMR & 32 \\
\hline Mortality (\%) & & 10 & & 8 & & 0 \\
\hline
\end{tabular}

TKIs: Tyrosine Kinase Inhibitors; CHR: Complete Hematological Response; CCyR: Complete Cytogenetic Response; MMR: Major Molecular Response; CMR: Complete Molecular Response Table 2: Adverse events and response depending on each TKIs 


\section{Discussion}

This study investigated the selection of TKI therapy in a real-world setting in Japan, given the lack of an established consensus. All patients diagnosed with CML prior to 2009 were inevitably started on imatinib, and 66\% remained on imatinib. The deep MMR treatment outcome achieved with imatinib, dasatinib, and nilotinib was $75 \%$, $84 \%$, and $71 \%$, respectively. Regardless the type of TKI (first or second generation), the treatment outcome was generally favorable, with failure to achieve MMR observed in $17 \%$ of patients receiving imatinib, $12 \%$ receiving dasatinib, and $0 \%$ receiving nilotinib. Patients receiving imatinib and dasatinib who failed to achieve MMR included which did not receive the adequate dosage because of adherence problems. Adherence may therefore be an important consideration in the real-world treatment of CML.

In the previous studies of the treatment of CML using TKIs, the IRIS [2], DASISION [8] and ENESTnd [9] trials, the discontinuation rate was $28 \%, 36 \%$, and $37 \%$, respectively. In our study, dasatinib had a slightly higher dose reduction rate of $40 \%$ and a switching rate of $20 \%$, mostly attributable to AEs (60\%), while the switching rate for nilotinib was extremely low (4\%). AEs related to nilotinib were primarily abnormalities in laboratory data, such as those indicative of thrombocytopenia and liver dysfunction, with fewer subjective symptoms. Thus, the low switching rate for nilotinib might be the result of more manageable AEs.

Interestingly, in the DASISION trial, dasatinib was the most commonly used second-line agent after the discontinuation of firstline treatment with imatinib. However, in our study, nilotinib was more commonly used as a second-line agent, potentially because the AEs associated with nilotinib were deemed by physicians to be more manageable. From the Japanese subgroup analysis in DASISION, pulmonary effusion was observed more frequently in Japanese patients than in the overall study population. Physicians may therefore avoid the use of dasatinib as second-line treatment because of the increased risk of pleural effusion in the Japanese population [9].

This study has some limitations, including its retrospective design and inclusion of a single facility. Additionally, the specific reason for second-line TKI selection by physicians was not captured.

Our data indicate that imatinib remains applicable as first-line treatment for some patients, with a satisfactory response rate and familiarity on the part of physicians, meaning that its associated AEs are more easily managed. Dasatinib was therapeutically effective, but of the incidence of associated pleural effusion is a challenge. Nilotinib was the most popular TKI for first- and secondline treatment at our facility, likely because of its associated AEs are more easily managed.

As described previously, the selection of TKI was based on each physician's judgement. We therefore plan to conduct head-to-head trials to establish a consensus about TKI use in the treatment of de novo CML.

\section{References}

1. Hochhaus A, Larson RA, Guilhot F, Radich JP, Branford S, et al. (2017) Long-Term Outcomes of Imatinib Treatment for Chronic Myeloid Leukemia. N Engl J Med 376: 917-27.

2. Hughes TP, Hochhaus A, Branford S, Müller MC, Kaeda JS, et al. (2010) Long-term prognostic significance of early molecular response to imatinib in newly diagnosed chronic myeloid leukemia: an analysis from the International Randomized Study of Interferon and STI571 (IRIS). Blood 116: 3758-65.

3. Marin D, Milojkovic D, Olavarria E, Khorashad JS, de Lavallade H, et al. (2008) European Leukemia Net criteria for failure or suboptimal response reliably identify patients with CML in early chronic phase treated with imatinib whose eventual outcome is poor. Blood 112: 4437-44.

4. Osborn M, Hughes T (2010) Managing imatinib resistance in chronic myeloid leukaemia. Curr Opin Hematol 17: 97-103.

5. Baccarani M, Saglio G, Goldman J, Hochhaus A, Simonsson B, et al. (2006) Evolving concepts in the management of chronic myeloid leukemia: recommendations from an expert panel on behalf of the European Leukemia Net. Blood 108: 1809-20.

6. Cancer Therapy Evaluation Program (CTEP) (2010) Common Terminology Criteria for Adverse Events (CTCAE) v4.0, USA.

7. Cortes JE, Saglio G, Kantarjian HM, Baccarani M, Mayer J, et al. (2016) Final 5-Year Study Results of DASISION: The Dasatinib Versus Imatinib Study in Treatment-Naïve Chronic Myeloid Leukemia Patients Trial. J Clin Oncol 34: 2333-40.

8. Hochhaus A, Saglio G, Hughes TP, Larson RA, Kim DW, et al. (2016) Long-term benefits and risks of frontline nilotinib vs imatinib for chronic myeloid leukemia in chronic phase: 5-year update of the randomized ENESTnd trial. Leukemia 30: 1044-54.

9. Fujisawa S, Nakamae H, Ogura M, Ishizawa K, Taniwaki M, et al. (2014) Efficacy and safety of dasatinib versus imatinib in Japanese patients with newly diagnosed chronic-phase chronic myeloid leukemia (CML-CP): Subset analysis of the DASISION trial with 2-year follow-up. Int J Hematol 99: 141-53. 


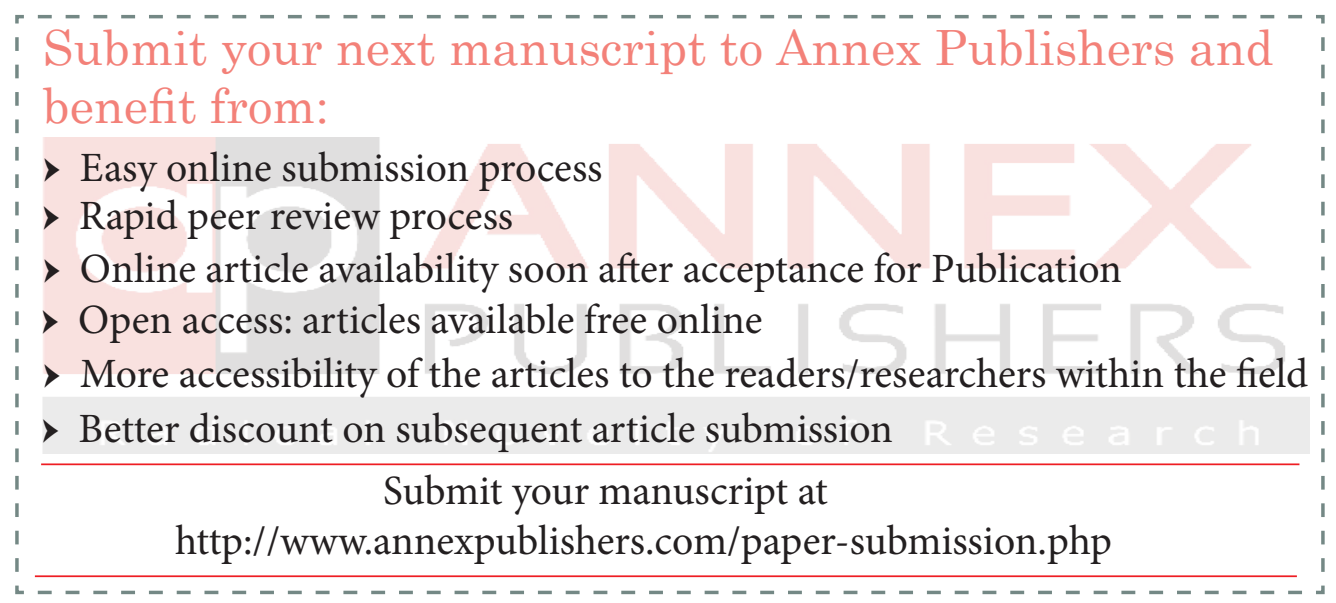

